\title{
Pulsations in hot subdwarf stars: recent advances and prospects for testing stellar physics
}

\author{
Stéphane Charpinet ${ }^{1}$, V. Van Grootel ${ }^{2}$, G. Fontaine ${ }^{3}$, P. Brassard ${ }^{3}$, \\ S.K. Randall ${ }^{4}$ and E. M. Green ${ }^{5}$ \\ ${ }^{1}$ CNRS, UPS-OMP, Université de Toulouse, Toulouse, France \\ email: stephane.charpinet@irap.omp.eu \\ ${ }^{2}$ Institut d'Astrophysique et de Géophysique, Université de Liège, Liège, Belgium \\ ${ }^{3}$ Département de Physique, Université de Montréal, Montréal, Canada \\ ${ }^{4}$ ESO, Garching bei München, Germany \\ ${ }^{5}$ Steward Observatory, University of Arizona, Tucson, AZ, USA
}

\begin{abstract}
The evolved, core helium burning, extreme horizontal branch stars (also known as hot B subdwarfs) host several classes of pulsators showing either p- or g-modes, or both. They offer particularly favorable conditions for probing with asteroseismology their internal structure, thus constituting arguably the most interesting seismic window for this intermediate stage of stellar evolution. G-modes in particular have the power to probe deep inside these stars, down to the convective He-burning core boundary where uncertain physics (convection, overshooting, semi-convection) is at work. Space data recently obtained with CoRoT and Kepler are offering us the possibility to probe these regions in detail and possibly shed new light on how these processes shape the core structure. In this short paper, we present the most recent advances that have taken place in this field and we provide hints of the foreseen future achievements of hot subdwarf asteroseismology.
\end{abstract}

Keywords. stars : oscillations, subdwarfs

\section{Pulsations in hot subdwarfs}

Hot subdwarfs refer to the hot and compact objects generally associated with intermediate (usually post-red giant) phases of stellar evolution (see, e.g., the general review of Heber 2009). They come in two main flavors with the B-type subdwarf (sdB) stars, at effective temperatures ranging from 22,000 and 40,000 K, and the hotter O-type subdwarf (sdO) stars having $T_{\text {eff }}$ above $\sim 40,000 \mathrm{~K}$ (Figure 1 ). Hot B subdwarfs are nowadays clearly associated with the extreme horizontal branch stars burning helium in their core, while the nature of sdO stars remains more fuzzy, this spectral type including most likely stars of various origins. Nevertheless, among the sdO stars, one should expect to find post-EHB objects on their way to the white dwarf cooling track, after core helium exhaustion.

Hot subdwarf asteroseismology has been developing following the discoveries of two classes of pulsating sdB stars (Kilkenny et al. 1997; Green et al. 2003; see again Figure 1) and the theoretical work that established the mechanism behind these instabilities (Charpinet et al. 1997; Fontaine et al. 2003) as well as the properties of the oscillation modes in this type of stars (Charpinet et al. 2000, 2002). Pulsations in this intermediate stage of stellar evolution involve rapid $p$-mode oscillations on timescales of a few minutes (the V361 Hya pulsators) or slow $g$-mode oscillations with periods of $1-4$ hours (the 


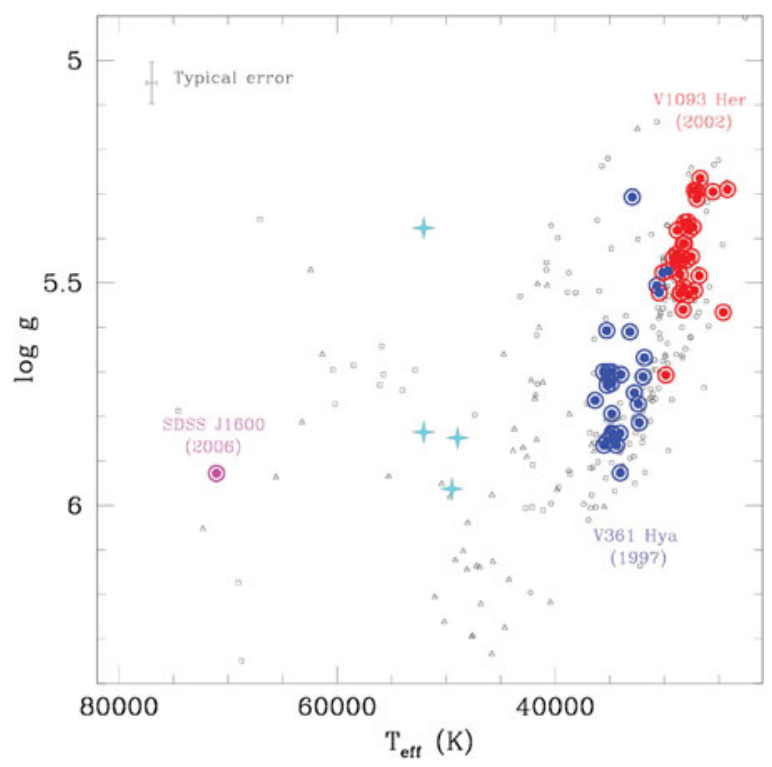

Figure 1. Distribution of hot subdwarfs in the $\log g-T_{\text {eff }}$ plane. Open circles show sdB stars, open squares are sdO stars, and open triangles represent helium-rich subdwarfs. The various classes of subdwarf pulsators are indicated (with the year of their discovery). The sdB stars host two classes of nonradial pulsators : the V361 Hya (in blue) and V1093 Her (in red) classes. Hot sdO pulsators are indicated in magenta (a star from the galactic field) and as cyan crosses for pulsating sdO's discovered in the globular cluster $\omega$-Cen. We point out that the sample shown in this figure is homogeneous in terms of the determination of the stellar atmospheric parameters from spectroscopy.

V1093 Her pulsators). In addition, a few stars are found to be hybrid pulsators showing both kind of modes simultaneously (Schuh et al. 2006). In all cases, the oscillations are caused by an opacity $(\kappa$-)effect involving iron-group elements and radiative levitation to enrich sufficiently the driving region in the stellar envelope. If iron is the prime element acting in this driving mechanism, it was shown later on that nickel also has an important contribution, in particular for defining the blue edge of the long period $g$-mode sdB pulsator instability region (Jeffery et al. 2006, 2007; Hu et al. 2009; Bloemen et al. 2014). We have to mention here that very recently Østensen et al. (2014) referred to the discovery of amplitude and phase modulations in a pulsating sdB star monitored with the Kepler satellite as the signature of "stochastic pulsations" in this star. But the term "stochastic" can be misleading here and the phenomenon should not be confused with the stochastic (convection induced) driving mechanism affecting Sun-like stars. Moreover, such modulations are not necessarily random and can possibly be linked to nonlinear resonant mechanisms affecting the pulsation modes of the star (see Zong et al. 2016, in preparation). For completeness, although these objects will not be discussed further in this short paper, we have to mention that hot subdwarfs also host pulsators in the very high temperature range where sdO stars are found (Figure 1). The first pulsating star of this type was discovered by Woudt et al. (2006) and shows very rapid oscillations $(P \sim 40-150 \mathrm{~s})$ associated with low-order $p$-modes. Surprisingly, this star remains so far the unique member of this class found among the field sdO stars, despite significant efforts to search for pulsations in such objects (Rodríguez-López et al. 2008). More pulsating sdO stars have however been found, but in the globular clusters $\omega$ Cen (Randall et al. 2011) and NGC 2808 (Brown et al. 2013). However, it is not clear if those cluster objects belong 
to the same class of pulsators as the star discovered by Woudt et al. (2006) because they do not seem to occupy the same region in the $\log g-T_{\text {eff }}$ plane (Figure 1). Theoretically speaking, it is thought that a mechanism similar to the one driving oscillations in sdB stars can excite the pulsations in sdO stars (Fontaine et al. 2008).

\section{Recent advances in hot B subdwarf asteroseismology}

\subsection{Quantitative asteroseismology}

The first quantitative asteroseismic inference of the fundamental properties of a sdB star came with the pioneering work of Brassard et al. (2001) on the $p$-mode pulsator $P G$ $0014+067$, using grids of envelope models. The technique was then vastly improved over the years with the development of sophisticated optimization tools and a new generation of stellar models. The typical outcomes from the asteroseismic studies of $p$-mode pulsators have been the determination of fundamental parameters - such as the mass, surface gravity, and hydrogen dominated envelope mass of the star - to unprecedented levels of precision (e.g., Charpinet et al. 2005; Van Grootel et al. 2013) and in some cases the determination of the internal rotation (e.g., Charpinet et al. 2008). These (still ongoing) efforts led to the seismic analysis of $15 \mathrm{sdB}$ pulsators so far, with interesting applications such as, for instance, inferring their mass distribution (Fontaine et al. 2012), a key information to understand the still unclear processes leading to the formation of sdB stars (see, Charpinet et al. 2011a; Geier 2015 and references therein). However, the core of these stars remained out of reach until the opportunity came to exploit the long period sdB pulsators whose $g$-modes penetrate deeper than the acoustic waves do (Charpinet et al. 2000). This became possible with the advent of space observations of pulsating sdB stars with CoRoT (Charpinet et al. 2010) and Kepler (Østensen et al. 2010, 2011). Presently, 18 sdB stars, among which most are $g$-mode pulsators, have ultra high precision photometry available and revealing extremely rich pulsation spectra. First attempts to probe their core have been made on only 3 of them (Van Grootel et al. 2010a,b Charpinet et al. 2011b). These preliminary results led to strong suggestions that the convective mixed core is significantly larger than expected from standard evolution models, thus proving the potential of these stars for investigating the structure and evolution of helium burning cores. Much still needs to be done on that subject however (see below).

One interesting new opportunity offered by such precise quantitative seismic inferences is to provide tests of the constitutive physics involved in stellar models. An example of such tests is illustrated in Figure 2 (see also Van Grootel et al. 2013). The idea here was to evaluate the impact of changing the iron abundance profile in the envelope of a sdB star from a nonuniform distribution derived by assuming diffusive equilibrium between radiative levitation and gravitational settling (the "standard" models) to profiles that either reduce the amount of levitating iron by a factor of 2 (test case 1) or 4 (test case 2), or that disable the expected effects of microscopic diffusion by assuming an homogeneous repartition of iron in solar proportion (test case 3). Because iron has a significant contribution to the mean Rosseland opacity, each case will generate models with different thermal structures and pulsation periods at given parameters, thus affecting the determination of the stellar properties derived from asteroseismology. However, since the object analyzed, the star NY Vir, is a pulsating sdB star in a close eclipsing binary system, precise independent measurements of the mass, radius, and surface gravity $(\log g)$ of the star are also available from spectroscopy and binary lightcurve analysis, meaning that the accuracy of the seismic solutions obtained for each case can also be tested. 
Impact of iron abundance profiles

(standard models : diffusive equilibrium)

Test case $1 \rightarrow$ levitating Fe reduced by a factor of 2
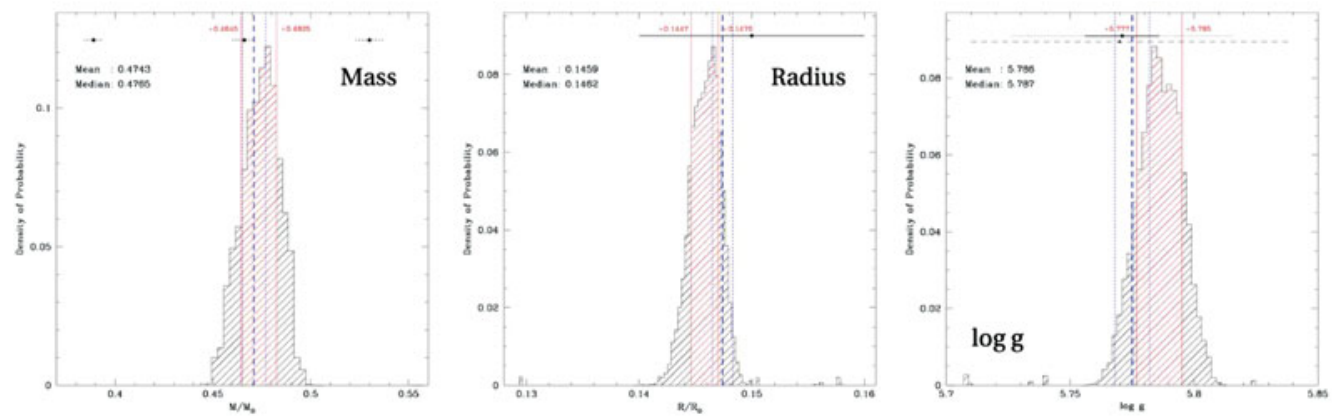

$\underline{\text { Test case } 2} \rightarrow$ levitating Fe reduced by a factor of 4
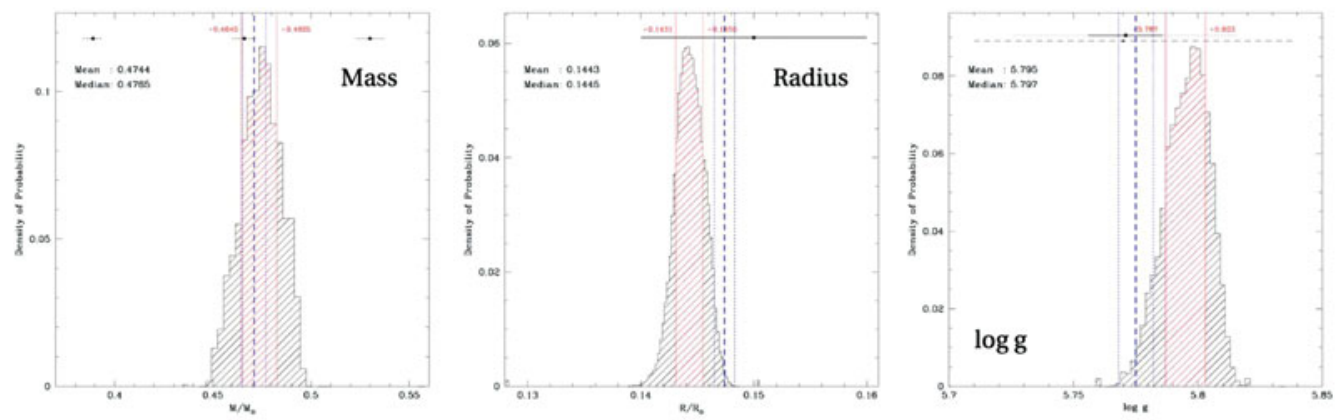

\section{$\underline{\text { Test case } 3} \rightarrow$ uniform iron in solar proportion (no diffusion)}
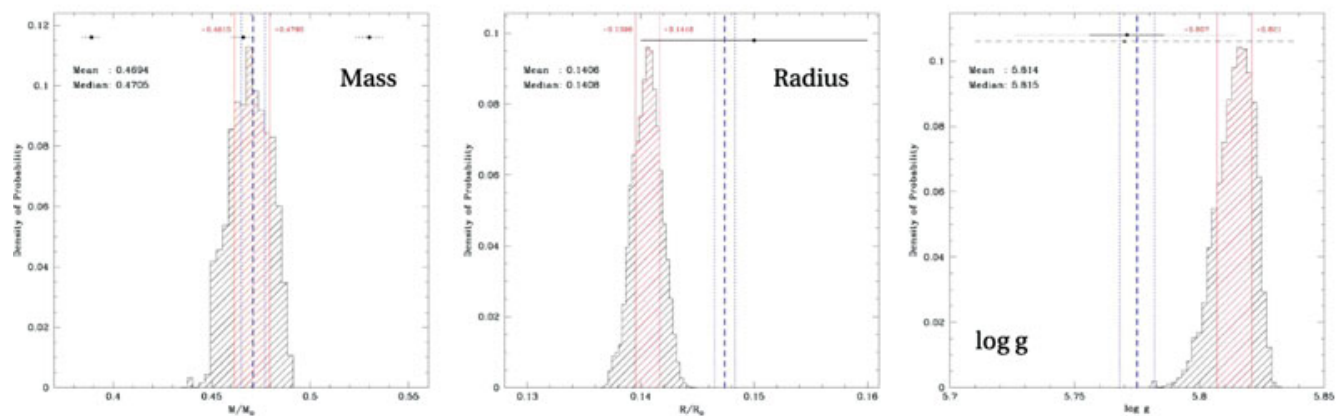

Figure 2. Determination of the stellar mass, radius, and $\log g$ of the star NY Vir from asteroseismology (histograms represent the density of probability distribution) using different sets of stellar models including : 1) a levitating amount of iron divided by 2 compared to the profile predicted by equilibrium between radiative levitation and gravitational settling (test case 1), 2) a levitating amount of iron divided by 4 (test case 2), and 3 ) a homogeneous distribution of iron in solar proportion (i.e., no diffusion; test case 3). The seismic results obtained for each case have to be compared to the seismic solution obtained with our standard models incorporating iron profiles in diffusive equilibrium (vertical blue dashed lines with the dotted lines indicating $1 \sigma$ errors). They also have to be compared with the independent measurements obtained from spectroscopy and from the analysis of the orbital lightcurve of this close eclipsing binary system. Clearly the solution with the standard models show the best overall consistency, while a stellar envelope having a solar homogeneous distribution for iron can be ruled out. 
Figure 2 shows that a very good consistency between all the independent measurements can be obtained with the standard models (assuming iron profiles in diffusive equilibium) while the seismic solution tend to diverge significantly (at least in terms of radius and $\log g$ ) for the other cases. Situations permitting this type of experiments, i.e., when some stellar parameters can be measured very precisely by other means than asteroseismology, are therefore extremely useful for testing indirectly some of the microphysics involved in stellar models.

\subsection{First glimpses on the internal rotation of sdB stars}

Asteroseismology can also, in principle, provide strong constraints on the internal dynamics (rotation) of a star when signatures, such as rotational splittings, can be identified in the pulsation spectra. For pulsating sdB stars, such opportunities have surprisingly remained far and few until very recently. In the pre-space observation era, only a few cases of sdB stars with seismic inferences of their internal rotation have been proposed, most notably with the analysis of PG 1336-018 (NY Vir, already discussed above, Charpinet et al. 2008) and Feige 48 (Van Grootel et al. 2008). Both objects were found to rotate rigidly and fairly rapidly (with periods of a few hours) in synchronization with the orbital motion of a close companion. Otherwise, most sdB pulsators monitored from the ground with modest time baselines (usually less than 2 or 3 weeks per campaign at best) were simply lacking such rotation signatures. Space observations, in particular with the Kepler mission, have now given us the reason why such signatures have been so few to be detected in these stars : most sdB pulsators appear to be very slow rotators that could simply not be resolved within the duration of typical ground based campaigns.

Based on Kepler data, the rotation rates of 4 more sdB stars in compact binary systems have been estimated, showing that unsynchronized systems where the sdB component rotates more slowly (with periods between 7.4 and 41 days) can also be found (Pablo et al. 2011, 2012; Østensen al. 2014). This opens up very interesting opportunities to test tidal synchronization timescales (and theories) with such binary systems. Indeed the rotation of sdB stars in close binaries most likely reflects the influence of tidal interactions with the companion. Isolated sdB stars, for their part, should not be affected by such effects and their rotation therefore mostly reflects their past history, i.e., the result of the secular evolution of angular momentum in their progenitors. Evidence is growing that these stars are very slow rotators with rotation periods of the order of weeks or even months (Baran et al. 2009, 2013; Charpinet et al. 2011; Foster et al. 2015; Peters et al. 2016, in preparation). Interestingly, such timescales can be connected to recent results from asteroseismology of red giants clearly indicating that the cores of red clump stars also rotate very slowly, with periods strikingly similar to those inferred for isolated sdB stars (see, e.g., Aerts 2015; Mosser et al. 2013 and references therein). This dynamical analogy strongly suggests that isolated sdB stars may have evolved from the same channel that produces most horizontal branch stars, that is through the standard red-giant evolution channel (thus possibly challenging the alternative merger scenario).

A connection can also be made with the results obtained over the years from white dwarf asteroseismology showing that white dwarfs typically rotates slowly as solid bodies with periods in the $\sim 5-60$ hour range. One can then notice that shrinking a sdB star (or red-clump stellar core) to the size of a typical white dwarf assuming total angular momentum conservation would indeed lead to the observed white dwarf rotation rates. This indicates that no more angular momentum is lost by stellar cores past the red-giant phase. Figure 3 provides a schematic summary of the overall emerging picture when considering the entire life of low mass stars. We can see that a typical main sequence star (say the Sun) must undergo very strong braking of its rotation prior reaching the 


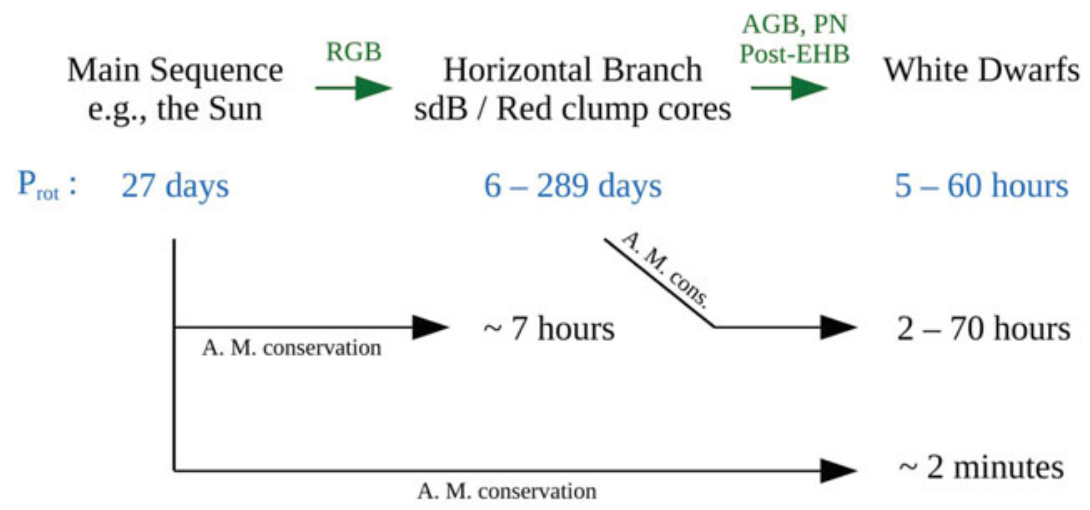

\begin{tabular}{|l|l|l|l|l|}
\hline Star & Type & Prot (h) & Jtot (kg.m2/s) & Jtot / Jsun \\
\hline Sun & Solar type & $\sim 648(27 \mathrm{~d})$ & $1.92 \times 10^{41}$ & $\mathbf{1}$ \\
\hline PG1159-035 & Pre-WD & $33.7(1.40 \mathrm{~d})$ & $\mathbf{1 . 2 5} \times \mathbf{1 0}^{\mathbf{3 9}}$ & $\mathbf{1 / 1 5 4}$ \\
\hline KIC08626021 & DB WD & $46.8(1.95 \mathrm{~d})$ & $\mathbf{6 . 5 4 \times 1 \mathbf { 1 0 } ^ { 3 8 }}$ & $\mathbf{1 / 2 9 4}$ \\
\hline GD165 & DA WD & $57.2(2.38 \mathrm{~d})$ & $\mathbf{4 . 8 9 \times 1 \mathbf { 1 0 } ^ { \mathbf { 3 8 } }}$ & $\mathbf{1 / 3 9 3}$ \\
\hline Isolated sdBs & sdB & $\sim 960(40 \mathrm{~d})$ & $7.60 \times 10^{38}$ & $1 / 253$ \\
\hline
\end{tabular}

Figure 3. Schematic view of the rotation period and angular momentum evolution throughout the main stages in the life of low mass stars. Typically measured rotation periods $\left(P_{\text {rot }}\right)$ are used for comparisons with expectations when assuming total angular momentum conservation. The bottom table gives the typical values for the total angular momentum (assuming rigid rotation) compared to the Sun. Clearly, angular momentum appears to be completely lost during and/or before the red giant phase, while nothing much happens afterward during the evolution.

horizontal branch, most probably during the red giant phase, in order to match the rotation rates measured in the late phases of evolution. We estimate that the total angular momentum of a typical main sequence star needs to be reduced by a factor $\sim 200-$ 400, thus providing an interesting quantitative constraint for identifying the mechanisms responsible for the braking.

\section{Prospects for probing He-burning cores}

The g-modes observed in the long period sdB stars offer an important advantage over the p-modes seen in fast sdB pulsators: they propagate much deeper inside the star than the shallower (mostly envelope) acoustic modes normally do. This property, which is classical in nondegenerate stars, opens the way to deep asteroseismic probing of the helium burning cores of extreme horizontal branch stars. The pioneering studies of Van Grootel et al. (2010a,b) and Charpinet et al. (2011b) have illustrated the strong potential of this approach to unravel some aspects of the structure of these cores. The latter is closely related to notoriously uncertain mixing mechanisms (convection, overshooting, semi-convection) that are at work to shape the core during this phase of stellar evolution.

The development of a partial mixing zone (often referred to as semi-convection) at the edge of the convectively mixed core at some point of the evolution is of particular interest. Stellar evolution codes usually describe this phenomenon with a prescription (see, e.g., Langer et al. 1985) providing a way to calculate the chemical stratification in that region, sometimes imposing smooth chemical gradients. But recent 3D 
hydrodynamical simulations from Mirouh et al. (2012) and Wood et al. (2013) suggest that such partial mixing zones could instead develop layered (stair-like) chemical stratifications. The question of the stability of such layers over long timescales and even their existence in real stellar core conditions remains largely open, however. Because $g$-modes in sdB stars propagate in the partial mixing zone when it develops at the core boundary, model calculations show that they are quite sensitive, through the so-called trapping effects, to any sharp chemical gradient that would eventually build up in that region. It should therefore be possible to assess whether semi-convection indeed produces layered stratifications as suggested by $3 \mathrm{D}$ simulations, or whether the chemical profiles in that region are smooth. We point out in this context, that the convincing identification of a sequence of trapped modes in the sdB star KIC 10553698A (Østensen et al. 2014) suggests, when compared to pulsation models, that sharp structures in the core region may be the cause of this trapping. A detailed quantitative seismic analysis of this star is however needed to draw firm conclusions on that highly promising case.

\section{Conclusion and general prospects}

Hot B subdwarf pulsators offer great opportunities for studying : 1) the structure and evolution of helium burning cores of low mass stars, 2) the physics of convection, semiconvection, and overshooting, and 3) the internal dynamics (rotation) of He-core burning stars in terms of both the secular evolution of angular momentum and the effects of tidal interactions in close binaries. The legacy of the Kepler mission is particularly important in this context with data available for more than $18 \mathrm{sdB}$ pulsators which have yet to be fully exploited through the current development of new generations of models dedicated to ultra high precision asteroseismic studies of these stars that is being done in parallel. Observationally speaking, strong prospects exist to benefit from the future NASA/TESS and ESA/PLATO missions to extend further our sample of very high precision data obtained from space for this kind of stars. This very positive context should trigger significant improvements in our understanding of stellar physics.

\section{Acknowledgement}

This work was supported in part by the Programme National de Physique Stellaire (PNPS, CNRS/INSU, France) and the Centre National d'Etudes Spatiales (CNES, France). Part of this work was performed using HPC resources from CALMIP (Grant 2015-P0205).

\section{References}

Aerts, C. 2015, Astronomische Nachrichten, 336, 477

Baran, A. S. \& Østensen, R. H. 2013, AcA, 63, 79

Baran, A., Oreiro, R., Pigulski, A., et al. 2009, MNRAS, 392, 1092

Bloemen, S., Hu, H., Aerts, C., et al. 2014, A\&A, 569, A123

Brassard, P., Fontaine, G., Billères, M., et al. 2001, ApJ, 563, 1013

Brown, T. M., Landsman, W. B., Randall, S. K., Sweigart, A. V., \& Lanz, T. 2013, ApJL, 777, L22

Charpinet, S., Fontaine, G., Brassard, P., et al. 1997, ApJL, 483, L123

Charpinet, S., Fontaine, G., Brassard, P., \& Dorman, B. 2000, ApJS, 131, 223

Charpinet, S., Fontaine, G., Brassard, P., \& Dorman, B. 2002, ApJS, 139, 487

Charpinet, S., Fontaine, G., Brassard, P., Green, E. M., \& Chayer, P. 2005, A\& $\&, 437,575$

Charpinet, S., Van Grootel, V., Reese, D., et al. 2008, $A \mathscr{E} A$, 489, 377

Charpinet, S., Green, E. M., Baglin, A., et al. 2010, A\&A, 516, L6 
Charpinet, S., Fontaine, G., Brassard, P., et al. 2011a, Nature, 480, 496

Charpinet, S., Van Grootel, V., Fontaine, G., et al. 2011b, A\&A, 530, A3

Fontaine, G., Brassard, P., Charpinet, S., et al. 2003, ApJ, 597, 518

Fontaine, G., Brassard, P., Green, E. M., et al. 2008, A\&GA, 486, L39

Fontaine, G., Brassard, P., Charpinet, S., et al. 2012, A\&A, 539, A12

Foster, H. M., Reed, M. D., Telting, J. H., Østensen, R. H., \& Baran, A. S. 2015, ApJ, 805, 94

Geier, S. 2015, Astronomische Nachrichten, 336, 437

Green, E. M., Fontaine, G., Reed, M. D., et al. 2003, ApJL, 583, L31

Heber, U. 2009, ARAA, 47, 211

Hu, H., Nelemans, G., Aerts, C., \& Dupret, M.-A. 2009, A\&A, 508, 869

Jeffery, C. S. \& Saio, H. 2006, MNRAS, 372, L48

Jeffery, C. S. \& Saio, H. 2007, MNRAS, 378, 379

Kilkenny, D., Koen, C., O'Donoghue, D., \& Stobie, R. S. 1997, MNRAS, 285, 640

Langer, N., El Eid, M. F., \& Fricke, K. J. 1985, A\&A, 145, 179

Mosser, B., Samadi, R., \& Belkacem, K. 2013, SF2A-2013: Proceedings of the Annual meeting of the French Society of Astronomy and Astrophysics, 25

Østensen, R. H., Silvotti, R., Charpinet, S., et al. 2011, MNRAS, 414, 2860

Østensen, R. H., Silvotti, R., Charpinet, S., et al. 2010, MNRAS, 409, 1470

Østensen, R. H., Reed, M. D., Baran, A. S., \& Telting, J. H. 2014, A\&̈A, 564, L14

Østensen, R. H., Telting, J. H., Reed, M. D., et al. 2014, A\&A, 569, A15

Mirouh, G. M., Garaud, P., Stellmach, S., Traxler, A. L., \& Wood, T. S. 2012, ApJ, 750, 61

Pablo, H., Kawaler, S. D., Reed, M. D., et al. 2012, MNRAS, 422, 1343

Pablo, H., Kawaler, S. D., \& Green, E. M. 2011, ApJL, 740, L47

Randall, S. K., Calamida, A., Fontaine, G., Bono, G., \& Brassard, P. 2011, ApJL, 737, L27

Rodríguez-López, C., Ulla, A., \& Garrido, R. 2008, Hot Subdwarf Stars and Related Objects, 392, 371

Schuh, S., Huber, J., Dreizler, S., et al. 2006, A\&SA, 445, L31

Van Grootel, V., Charpinet, S., Fontaine, G., \& Brassard, P. 2008, A\&A, 483, 875

Van Grootel, V., Charpinet, S., Fontaine, G., et al. 2010a, ApJL, 718, L97

Van Grootel, V., Charpinet, S., Fontaine, G., Green, E. M., \& Brassard, P. 2010b, A\& A, 524, A63

Van Grootel, V., Charpinet, S., Brassard, P., Fontaine, G., \& Green, E. M. 2013, A\&\&A, 553, A97

Wood, T. S., Garaud, P., \& Stellmach, S. 2013, ApJ, 768, 157

Woudt, P. A., Kilkenny, D., Zietsman, E., et al. 2006, MNRAS, 371, 1497 